\title{
La creación y uso de programas de educación ambiental es un elemento clave para la implementación efectiva de un sistema de gestión ambiental
}

\author{
Judith Elena Camacho', Ana Graciela Lancheros² \\ 1. Maestría en Medio Ambiente y Desarrollo. Docente Investigadora del Programa de Bacteriología y Laboratorio Clínico de \\ la Universidad Colegio Mayor de Cundinamarca Bogotá-Colombia \\ 2. Maestría en Modelos de Enseñanza Problémica. Docente Investigadora del Programa de Bacteriología y Laboratorio \\ Clínico de la Universidad Colegio Mayor de Cundinamarca Bogotá-Colombia. \\ Correspondencia: jelenacamacho@hotmail.com,agld2001@yahoo.es
}

Recibido 26-03-08 Aceptado 15-04-08

\begin{abstract}
Resumen
La creación y uso de programas de educación ambiental es un elemento clave para la implementación efectiva de un sistema de gestión ambiental. El programa debe describir como se alcanzarán las metas de la organización, responsables, cronograma de actividades, estrategias a seguir, inversiones requeridas y un sistema de registro. En este artículo se presentan los resultados del proyecto investigación "Validación del modelo sobre el manejo actual de los desechos generados en el área de laboratorios e instituciones prestadoras de salud". A las industrias que participaron en este proyecto investigativo se les aplicó un modelo elaborado por el Programa de Bacteriología de la Universidad Colegio Mayor de Cundinamarca; el cual se usa para evaluar el sistema de gestión ambiental y el manejo de los desechos de la misma Universidad y de una institución Prestadora de servicios de la Universidad. Además, se diseñó un programa educativo como soporte a la gestión ambiental en las industrias visitadas. Teniendo en cuenta los resultados, se considera que uno de los puntos de control críticos es la capacitación, donde la Universidad puede apoyar y retroalimentar la investigación en aspectos relacionados con la problemática ambiental y establecer vínculos entre la industria y la academia.

Palabras clave: educación ambiental, gestión ambiental, industria alimenticia, manejo de desechos, residuos sólidos.
\end{abstract}

\begin{abstract}
:
The creation and use of environmental education programs is a key element for the effective implementation of an environmental management system

The creation and use of programs of environmental education are a key element for the effective implementation of a system of environmental management. The program must describe the goals of the
\end{abstract}


organization, people in charge, timeline of activities, strategies to follow, required investments, and a recording system. This article shows the results of the investigation "Validation of the Model on Actual Handling of Waste Generated in Laboratories and Health Institutions". A model designed by the Program of Bacteriology of the University Colegio Mayor de Cundinamarca was applied to the industries involved in this research project; which was used to evaluate the environmental management system and waste management of the University itself and of the health institution of the University. Besides, a program was designed to support the environmental management in these industries. With these results, it is considered that one of the critical control posts is training, which the University can support and give feedback to the investigation in aspects related to environmental problems, establishing bonds between the industry and the university.

Key Words: environmental education, environmental management, food industry, waste handling, solid wastes.

\section{Introducción}

La educación ambiental requiere dos soportes fundamentales para lograr sus objetivos: de una parte la investigación, que aporta el conocimiento de las condiciones socio-ambientales del entorno, sus riquezas y potencialidades y, de otra, la participación ciudadana, que le permite orientar la formación de individuos y colectivos para la participación en procesos de gestión. En este sentido la investigación es un pilar fundamental para el avance y consolidación de la educación ambiental.

En este estudio, se analizó el cumplimiento de la normatividad en la temática relacionada con el manejo de desechos, mediante la aplicación del modelo elaborado para los laboratorios de práctica del Programa de Bacteriología e la Institución Prestadora de Servicios (IPS) de la Universidad Colegio Mayor de Cundinamarca (UCMC), con aplicación a instituciones de características similares en Bogotá, aportando herramientas conceptuales y metodológicas en el tema de educación ambiental.

Las instituciones participantes en el desarrollo de la investigación destacan la importancia de la conservación del medio ambiente y de desarrollar programas que permitan establecer vínculos entre la industria y la academia, y así apoyar y retroalimentar la investigación en aspectos que favorezcan el desarrollo de programas en gestión y educación ambiental.

\section{Metodología}

En la realización de este estudio se tuvo en cuenta las siguientes fases:

FASE I. Establecimiento de las actividades a valorar en base al modelo de manejo de desechos diseñado para los laboratorios de práctica e IPS de la UCMC y consideradas dentro de los parámetros de la gestión ambiental.

Actividad 1. Conocer las etapas que conforman el plan anual de manejo interno de los desechos.

Objetivo: controlar las labores de generación, separación, recolección, almacenamiento y el tratamiento en tanto el desecho permanezca dentro del servicio.

Actividad 2. Definición de las políticas ambientales y control de la generación de desechos desde su origen.

Objetivo: servir de soporte a la prevención y contaminación ambiental.

Actividad 3. Legislación actual sobre manejo de desechos.

Objetivo: cumplir la legislación vigente

Actividad 4. Técnicas apropiadas de tratamiento, almacenamiento y disposición final al total de desechos generados.

Objetivos: conocer y aplicar adecuadamente los procesos de tratamiento, almacenamiento y 
disposición final sobre los desechos generados.

Actividad 5. Aproximación empresarial.

Objetivo: concientizar y sensibilizar sobre la problemática ambiental del manejo de desechos y la relación causa efecto del manejo interno de los desechos sólidos sobre el medio externo.

Actividad 6. Diseño de un sistema de registro.

Objetivo: diseñar un sistema de registro que asegure la calidad de los procesos relacionados con el manejo de desechos.

\section{Actividad 7. Implementación de la cultura del reciclaje}

Objetivo: aplicar programa de reciclaje en la institución.

FASE II. Diseño de subproyectos y visita a industrias de alimentos.

FASE III. Valoración y análisis de las etapas propuestas como puntos de control.

FASE IV: Diseño del Programa de Educación Ambiental.

\section{Resultados}

FASE I. Establecimiento de las actividades a valorar en base al modelo de manejo de desechos diseñado para los laboratorios de práctica e IPS de la UCMC.

Las actividades mencionadas en la metodología, se establecieron con base al modelo del manejo de desechos diseñado, el cual tiene en cuenta el objetivo de la política de residuos y la planificación que involucra dos etapas básicas: identificar las necesidades actuales y una vez, éstas se hayan identificado determinar qué hacer para remediar los aspectos críticos y administrar eficientemente los diversos flujos de residuos.

Además, teniendo en cuenta los aspectos técnicos que involucra la política para la gestión de los residuos, se crearon líneas de acción en las diferentes etapas que componen la gestión de los residuos: generación, almacenamiento, recolección, aprovechamiento, tratamiento y disposición final (1). Dentro de las actividades definidas se incluyeron los parámetros de gestión ambiental: filosofía ambiental, cumplimiento de la legislación, aproximación empresarial y diseño de un sistema de registro (2).

\section{FASE II. Diseño de subproyectos y visita a industrias} de alimentos.

Dentro de las problemáticas ambientales del manejo de residuos establecidas en la política para la gestión integral de residuos, promulgada por el Ministerio del Medio Ambiente (MMA) en 1997, se encuentran las prácticas inadecuadas de disposición final, el bajo desarrollo institucional del sector y al falta de educación y participación ciudadana en el manejo ambiental de residuos (1). El diseño de los subproyectos se realizó teniendo en cuenta las problemáticas ambientales mencionadas, destacándose que el sistema universitario estatal no forma profesionales que se incorporen a las actividades de gestión de residuos, lo cual nos llevó a involucrar a la industria. Este sector seleccionado correspondió al alimentario, que según estudios desarrollados por el Departamento Nacional de Planeación (DNP), es el que más aporta a la contaminación industrial en Colombia (3).

El desarrollo del trabajo en las industrias de alimentos en Bogotá, se hizo dentro del proyecto institucional "Validación y Divulgación del Modelo de Manejo de Desechos", como complemento del Proyecto institucional "Diagnóstico del manejo actual de los desechos generados en el laboratorio central, área de laboratorios y la IPS de la universidad (4).

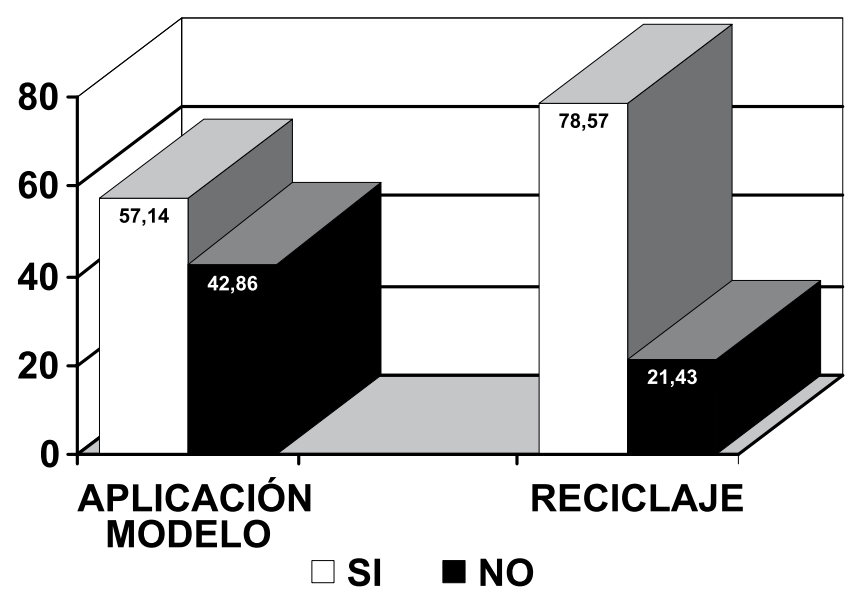

Figura 1. Lineamientos para el manejo adecuado de desechos. 
FASE III. Valoración y análisis de las etapas propuestas como puntos de control.

Las actividades consideradas dentro de los parámetros de la gestión ambiental en cada una de las industrias, muestran los siguientes valores:

Actividad 1: conocer las etapas que conforman el plan anual de manejo interno de los desechos

En el control de las labores de generación, separación, recolección y tratamiento dedesechos se encontró que el 57,14\% de las empresas visitadas tienen y aplican un modelo que da los lineamientos para el manejo adecuado de desechos, incluyendo el reciclaje como una de las estrategias de manejo, Figura 1, lo cual se promulga en la política de gestión integral de residuos del MMA (1). En las empresas se conoce la legislación vigente, pero no se realiza el proceso de seguimiento, validación y verificación de la aplicación correcta del modelo, que sólo se reporta en un $21,43 \%$.

Como metas propuestas para lograr cumplir esta actividad se plantean:

- Minimizar la generación de desechos.

- Supervisar el buen desempeño de las labores realizadas por la empresa de aseo municipal quien se encarga de la recolección, almacenamiento, transporte y tratamiento de los desechos sólidos.

- Establecer metas concretas a corto y mediano plazo, para la reducción de la cantidad de residuos generados y de una buena separación y tratamiento de los desechos.

- Contemplar un programa de reutilización y reciclaje de los residuos.

- Incluir un plan de contingencia para enfrentar las situaciones de emergencia.

Se detectó, que aunque en Colombia el Ministerio de Protección Social, el Ministerio del Medio Ambiente, Vivienda y Desarrollo y el Departamento Administrativo del Medio Ambiente han expedido una legislación, que se puede considerar la más completa de América Latina, no todas las empresas aplican la totalidad de estas normas, ya sea por desconocimiento de las mismas o por carencia de recursos para aplicarlas.

Como soporte a la prevención y contaminación ambiental es importante que las empresas definan una

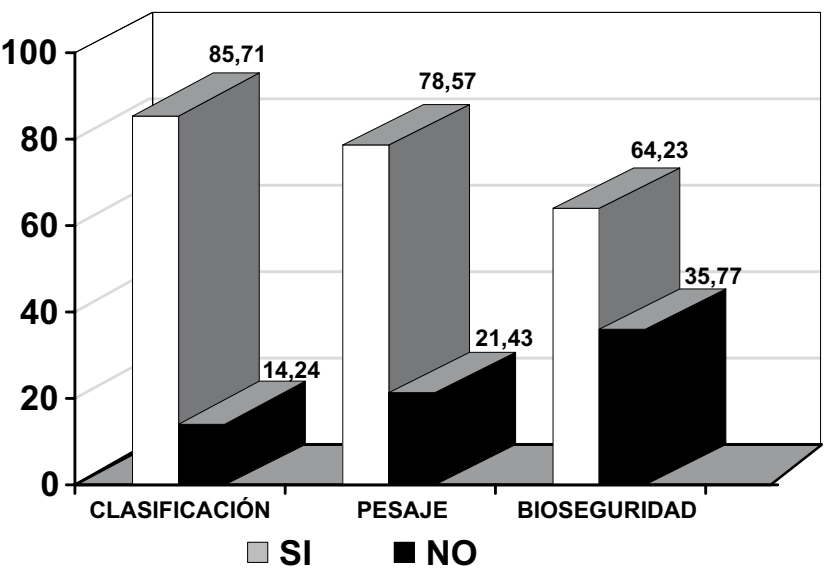

Figura 2. Categorías que definen la filosofía ambiental.

filosofía ambiental (2), a fin de controlar la generación de desechos desde su origen, para lo cual se tienen en cuenta las categorías clasificación, pesaje y bioseguridad.

\section{Actividad 2: Definir la Filosofía ambiental: controlar} la generación de desechos desde su origen.

Se puede destacar que el $85.71 \%$ de las empresas visitadas clasifican selectivamente el 100\% de los desechos generados, el 78,57\% realiza una determinación de la cantidad de desechos generados por peso, se incluye como una de las estrategias de manejo de desechos el uso de código de colores en algunas empresas $42.86 \%$ y el $64.23 \%$ aplica las normas de bioseguridad correspondiente, incluyendo planes de emergencia para prevenir accidentes 57,14\%.

Como metas propuestas para lograr cumplir esta actividad se proponen:

- Separar y clasificar los desechos en la fuente.

- Reducir la producción de desechos en la fuente.

- Reducir el riesgo de contaminación por manejo inadecuado de desechos.

- Clasificar la totalidad de los desechos generados.

- Desarrollar actividades de diagnóstico sobre manejo desechos.

- Determinar el tipo de desechos generados en un su totalidad.

- Disminuir la cantidad de desechos generados en un porcentaje establecido.

Actividad 3. Cumplir la legislación actual sobre manejo de desechos. 
Actividad 4. Aplicar técnicas apropiadas de tratamiento, almacenamiento y disposición final al total de desechos generados.

Como soporte del conocimiento y cumplimiento de la legislación vigente, el 50\% de las empresas visitadas posee un manual sobre manejo de desechos, donde se incluyen los parámetros exigidos para una correcta gestión de desechos y se cumplen los objetivos de la política

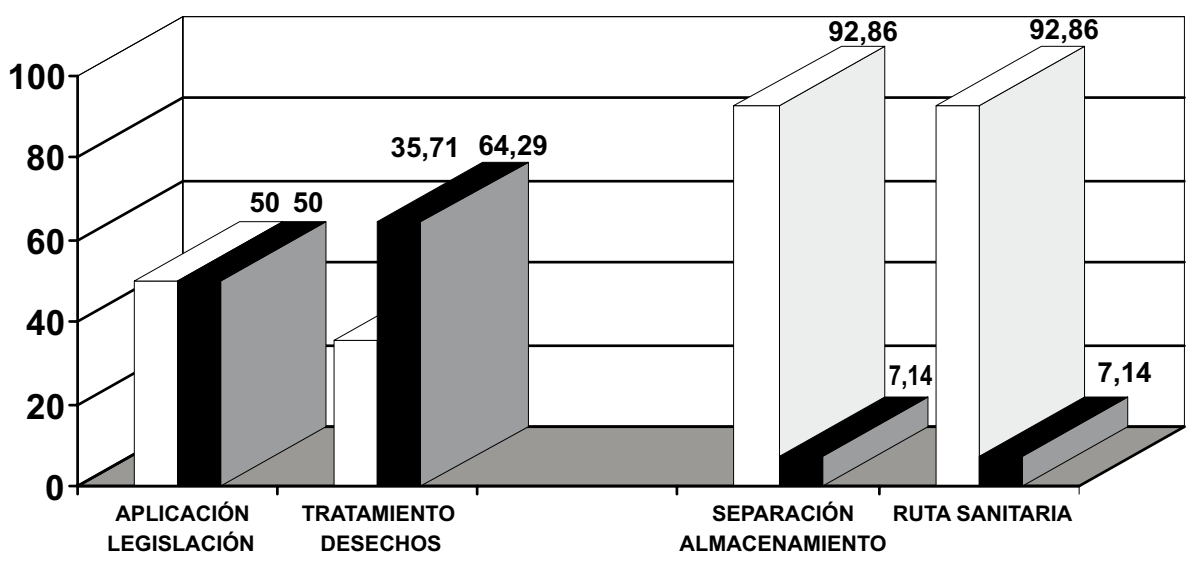

$\square$ SI $\square$ NO

Figura 3. Categorías relacionadas con la gestión de desechos. de residuos dada bajo principios del desarrollo sostenible, fundamentada en tres presupuestos: la minimización del impacto ambiental negativo que causan los residuos, el crecimiento económico y el mejoramiento de la calidad de vida de la comunidad en general. Además así como en la política de gestión de residuos se tiene en cuenta los residuos sólidos no peligrosos y los residuos sólidos peligrosos, involucrando los semisólidos cuando éstos no son vertidos a las fuentes de agua (1).

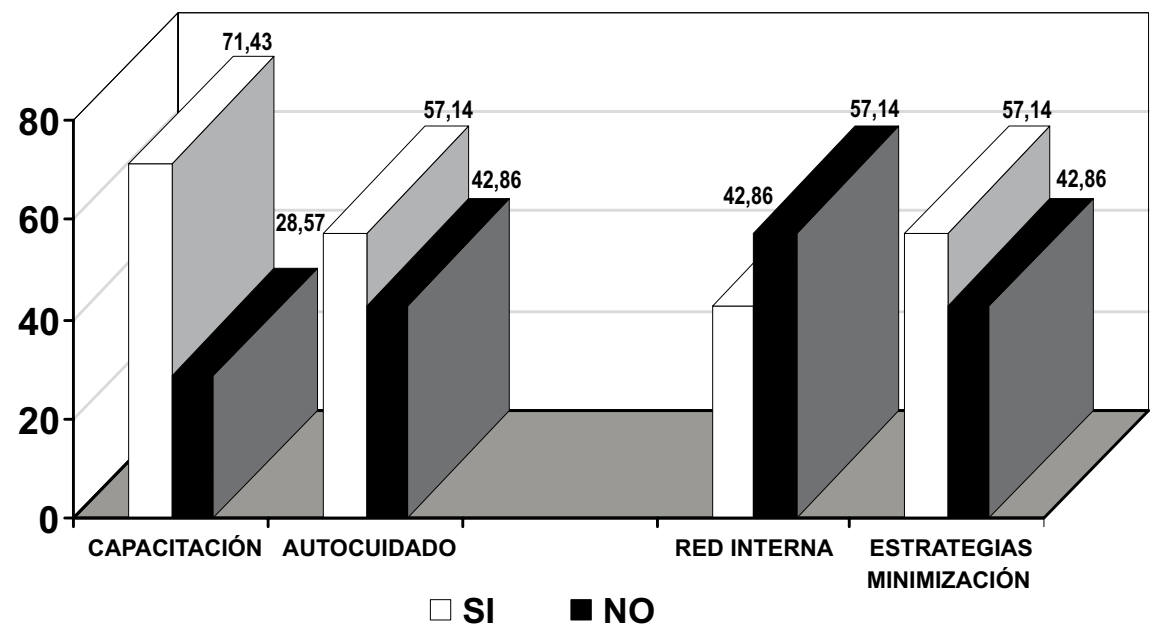

Con respecto al conocimiento y aplicación adecuada de los procesos de tratamiento, almacenamiento y

Figura 4. Aproximación empresarial. disposición final de los desechos generados, sólo el 35,71\% de las empresas visitadas realiza tratamiento in situ. Con respecto a las etapas de separación y almacenamiento, así como a la aplicación de una ruta sanitaria para el manejo de desechos, las empresas visitadas aplican estos parámetros en un 92,86\% Figura 3, lo cual se tiene en cuenta en los aspectos técnicos que la política para la gestión de residuos involucra, en las diferentes etapas que componen la gestión de los residuos: generación, almacenamiento, recolección, aprovechamiento y disposición final (1).

Como metas propuestas para lograr cumplir esta actividad se proponen:

- Dar a conocer la legislación a todo el personal.

- Establecer mecanismos de actualización sobre legislación vigente, sea por carteles o periódicos institucionales.

- Hacer el tratamiento adecuado para los diferentes tipos de desechos, según modelo sobre manejo de desechos.

- Realizar el almacenamiento inicial, intermedio y final apropiado de acuerdo al tipo de desecho.

- Establecer actividades de reciclaje sobre los desechos sólidos generados.

- Aplicar la ruta sanitaria apropiada.

- Entregar los desechos correctamente a la ruta externa.

- Suministrar y usar elementos de barrera a todo el personal que maneja desechos.

\section{Actividad 5. Aproximación empresarial}

Para que haya en las empresas una mayor sensibilización sobre la problemática ambiental del manejo de desechos y la relación causa efecto del manejo interno de los desechos 
sobre el medio externo, los indicadores utilizados para esta valoración mostraron que el $71,43 \%$ de las empresas visitadas presentan planes de capacitación sobre manejo de desechos, incluyendo legislación, técnicas de separación y tratamiento, así como sobre normas de autocuidado, 57.14\%, Figura 4.

Lo encontrado en las diferentes empresas se apoya en la política de gestión de residuos y en consecuencia la minimización de las basuras contribuye a conservar y reducir la demanda de recursos, disminuir el consumo de energía, preservar los sitios de disposición final y reducir sus costos, la contaminación ambiental al disminuir los residuos que van a los sitios de disposición final o que simplemente son dispuestos en cualquier sitio, contaminando el ambiente (1).

En las empresas visitadas la parte ambiental está incluida en todas las políticas institucionales, destacándose la creación de una red interna responsable del manejo de desechos en un $42.86 \%$, con lo cual se pretende minimizar la generación de desechos $57,14 \%$, soportados en el aprovechamiento y cultura del reciclaje, así como en la cultura del autocuidado, viendo estos lineamientos como estrategias empresariales para disminuir costos y generar ganancias. El aprovechamiento debe realizarse siempre y cuando sea económicamente viable, técnicamente factible y ambientalmente conveniente (1).

Como metas propuestas para lograr cumplir esta actividad se tienen:

- Desarrollar un sistema de sensibilización así como un mejoramiento continuo y trabajo en equipo, con base en la autogestión y autoevaluación institucional.

- Aplicar técnicas de aprovechamiento ya sea de manera directa o como resultado de procesos de tratamiento, reutilización, reciclaje, producción de bioabonos, compostaje, generación de biogas, incineración con producción de energía, aplicación de biotratamientos o técnicas de biorremediación, entre otros (5).

- Promover los incentivos propuestos en la política de gestión de residuos, en especial económicos y tributarios.

- Promover el uso de tecnologías limpias (producción más limpia).
FASE IV: Diseńo del Programa de Educación

\section{Ambiental.}

El programa de educación ambiental sobre manejo, recolección, reciclaje, transporte y disposición final de desechos generados en industrias de alimentos en Bogotá, se propone desarrollar, basado en la Enseñanza Problémica, utilizando principios como la situación problémica, la asimilación conciente y el trabajo independiente mediante ejercicios participativos e instrumentos metodológicos y técnicos como los que se presentan en el contenido de este programa (6).

Con este programa se pretende aportar herramientas conceptuales y metodológicas sobre la educación ambiental y su papel relevante en la gestión integral de residuos $(1,7)$. Vemos que muchas personas no comprenden cuán estrechos son los vínculos entre las actividades humanas y el medio ambiente, porque carecen de información acertada o suficiente. La educación ambiental no es un fin en sí misma, debe concentrarse en acciones estratégicas de participación y comunicación, principios establecidos en la política de gestión ambiental.

Ubicación del proyecto: Universidad Colegio Mayor de Cundinamarca e Industrias de alimentos.

Tipo de proyecto. Programa de educación ambiental El programa de educación ambiental propuesto parte de la actualización y elaboración de un diagnóstico del sector industrial alimentario para precisar el área de influencia del programa y las características de la comunidad a la cual se dirige, incluyendo la comunidad universitaria y los trabajadores de las industrias de alimentos, mediante un proceso de enseńanza problémica que involucra los temas de educación, comunicación, participación e investigación, basados en las categorías: trabajo, contradicción, problema entre otros (6).

Este programa pretende optimizar la participación de todos los actores involucrados en la gestión de residuos para cumplir tareas de asesoramiento, coordinación, liderazgo, evaluación, monitoreo y control con miras a provocar impactos positivos en la calidad ambiental del sector, fortaleciendo vínculos universidad-empresa. 


\section{Conceptos:}

Desarrollo sostenible: tal como lo definen las Naciones Unidas, es aquel que permite mejorar la calidad de vida humana sin rebasar la capacidad de carga de los ecosistemas que la sustentan. Es decir, aquel que satisface las necesidades del presente sin comprometer la capacidad de las generaciones futuras para satisfacer las propias (7).

Residuo o desecho: es cualquier objeto, material, sustancia, elemento o producto, que se encuentra en estado sólido o semisólido o es un líquido o gas contenido en recipientes o depósitos cuyo generador descarta, rechaza o entrega, porque sus propiedades no permiten usarlo nuevamente en la actividad que lo generó o porque la legislación o la normatividad vigente así lo estipula (1).

Generador de sectores productivos y de servicios: es responsable del manejo integral de residuos sólidos acorde con los programas de producción limpia que debe incluir sistemas de gestión ambiental para los residuos internos del proceso productivo y para los residuos post consumo de sus productos (1).

Gestión integral de residuos: es el término aplicado a todas las actividades asociadas en el manejo de los diversos flujos de residuos dentro de la sociedad y su meta es administrarlos de una forma compatible con el medioambiente y la salud pública (1).

Participación ciudadana: permite orientar la formación de individuos y colectivos para la participación en procesos de gestión (8).

Educación para la gestión ambiental: debe ser entendida no solo como el conjunto de los elementos pedagógicos formales que hacen referencia a los recursos naturales, sino como una adecuación pedagógica que logre cambiar las actitudes de la población hacia el entorno que las circunda (9).

1. Descripción y obras propuestas: incluye un sistema de seguimiento, validación y verificación del modelo elaborado sobre manejo de desechos, una clasificación de desechos, una determinación de cantidad de desechos, una evaluación y separación en la fuente, recolección, almacenamiento, transporte, tratamiento, disposición final, re-utilización y reciclaje de los desechos.

2. Estructura organizativa: creación de un comité encargado de elaborar el plan anual de manejo de residuos o desechos y de vigilar y controlar el buen desarrollo y funcionamiento del sistema. Con el objetivo de dar a conocer los parámetros que se deben tener en cuenta en un manejo adecuado de éstos, involucrando la importancia de la minimización en la generación de los mismos, así como la actividad del reciclaje y la conservación y mejoramiento del medio ambiente y la calidad de vida de la comunidad.

3. Objetivos: incrementar la seguridad, integrar la educación ambiental con la gestión ambiental y mejorar la calidad del ambiente disminuyendo la contaminación.

4. Población sujeto: comunidad universitaria, y personal de entidades externas de carácter industrial.

5. Estrategias.

5.1. Capacitación: conferencias de socialización, sobre la forma como se están manejando los desechos en cada una de estas áreas y cómo se pueden mejorar los procesos, mediante realización de talleres con aplicación de instrumentos de validación.

5.2. Determinación de puntos críticos de control en cada etapa del proceso de manejo de desechos, determinación de acciones correctivas.

5.3. Aplicación de los parámetros contenidos en el modelo elaborado sobre manejo de desechos en la Industria: realización trabajos de campo que involucre a la comunidad universitaria y al sector industrial, así como actividades de asistencia técnica y consultoría ambiental, enfocándose en la producción limpia, minimización de residuos, reciclaje, biotransformación de los mismos por acción de microorganismos y conocimiento de la legislación existente sobre manejo de residuos o desechos.

6. cciones del programa educativo:

Las acciones se encuentran formuladas en la Tabla No.1.

7. Instrumentos de trabajo:

- Diagnósticos ambientales: encuestas, entrevistas, lista de chequeo, matriz valorativa y observación directa.

- Análisis y priorización de problemáticas ambientales.

- Formulación, seguimiento y evaluación del proyecto.

- Informes de avance, registros, Indicadores de gestión, evaluación de la actividad. 
Tabla No. 1. Acciones del programa educativo

1. Conocimiento de los diferentes riesgos que pueden representar el manejo inapropiado de los residuos contaminados.

2. Forma segura de manipulación y manejo de residuos.

3. Características, manejo y limpieza de recipientes.

4. Procesos de clasificación y separación selectiva de residuos.

5. Uso adecuado, conservación y limpieza de elementos de protección.

6. Técnicas apropiadas para labores de limpieza y utilización segura de implementos de aseo.

7. Reporte de accidentes como derrames, filtraciones con fluidos, lixiviados o material contaminado.

8. Conceptos básicos sobre preparación de desinfectantes, detergentes y sus aplicaciones.

9. Procedimientos para la atención inmediata de accidentes, en la recolección y manejo de residuos, especialmente contaminados.

10. Aplicación de los conceptos básicos del autocuidado en las actividades de recolección, manejo y disposición de residuos (lavado de manos, etc.)

Fuente: Elaborado por los autores

8. Definición de actividades: intra y extramurales:

8.1 Intramurales: conferencias de socialización, realización de seminario-taller, las temáticas a trabajar son: comunicar el plan maestro, el modelo sobre manejo de desechos y la legislación, enseñar a manejar los registros necesarios en el manejo de desechos, trabajo de campo en el área donde aplica el modelo, realización de talleres prácticos con lluvias de ideas, realización de diagnósticos, elaboración de carteleras relacionadas con el tema, publicación de artículos en los medios de información interno, realización de jornadas y actividades de reciclaje, desarrollo de proyectos de investigación utilizando tecnologías limpias.

8.2 Extramurales: conferencia sobre manejo de desechos, incluyendo los parámetros de generación, caracterización, tratamiento, almacenamiento, recolección y transporte, disposición final, legislación, documentación y planes de contingencia sobre el manejo adecuado de desechos.

8.3 Red interinstitucional de intercambio de información y tecnología: diseño periódicos institucionales escritos $\mathrm{y}$ virtuales, carteleras institucionales y un sistema automatizado que garantice la comunicación en línea y genere información oportuna y confiable sobre el manejo de residuos o desechos. Sistema de información: crear un órgano de divulgación masiva destinado a informar y motivar sobre los programas a realizar y los logros obtenidos.

8.4 Informes de avance: diseñar un sistema de registros de evaluación de las actividades realizadas, mediante indicadores de gestión.
Identificar y convocar a instituciones existentes en la zona de acuerdo con su capacidad de gestión para realizar un trabajo interinstitucional: crear página web del programa y acceso a bancos más amplios de tecnología e información.

9. Inversiones requeridas: suministro de elementos de bioseguridad y seguridad industrial a todo el personal (elementos de prevención de incendios), mejoramiento de la ruta interna y señalización, así como de todo el soporte logístico para el funcionamiento correcto del programa, además involucrar las nuevas tecnologías de punta en comunicación.

\section{Discusión}

$\mathrm{La}$ industria es una de las principales fuentes de contaminación ambiental en el país. La industria nacional se ha apropiado del entorno natural para transformarlo en un sistema de disposición de residuos de costo mínimo. Adicionalmente a los residuos industriales convencionales que se descargan de manera continua a los cuerpos de agua como materias orgánicas, o a la atmósfera como partículas y monóxido de carbono, la descarga de sustancias y residuos tóxicos y peligrosos al entorno natural es creciente (3).

Los parámetros tenidos en cuenta para el desarrollo del programa educativo y la gestión ambiental en las industrias de alimentos estudiadas incluyen la aplicación y elaboración de un modelo, la definición de una filosofía ambiental, cumplimiento de la legislación actual sobre el manejo de desechos, aplicación de técnicas apropiadas de tratamiento, almacenamiento y disposición final de 
los desechos generados, la aproximación empresarial, el diseño de un sistema de registro, la implementación de la cultura del reciclaje y la comunicación (2).

La investigación formativa con el desarrollo de subproyectos de investigación en la Industria de alimentos, en el proyecto de investigación "Divulgación y Validación del Modelo de Manejo de Desechos", permitió establecer que los lineamientos tenidos en cuenta en la elaboración del modelo de manejo de desechos pueden ser aplicados a nivel industrial.

La ejecución de estos subproyectos permitió enriquecer y mejorar tanto el modelo propuesto, como ver las debilidades y fortalezas que tiene la industria alimentaria para el manejo de desechos, ya que según estudios desarrollados por el Departamento de Planeación Nacional (DNP) es una de las industrias que más aporta en la contaminación industrial en Colombia, generando una problemática ambiental (3).

Las empresas visitadas en general cumplen con la mayoría de los parámetros trabajados, pero con respecto a la existencia de un sistema de registro se ve un vacío en su diseño y aplicación. La importancia del diseño de un sistema de registro y su aplicación permite asegurar la calidad de los procesos relacionados con la gestión correcta de residuos.

El sistema de registro debe incluir registro diario de origen, cantidad, tipo de desecho producido, tratamiento, transporte y disposición final, de entrega de elementos de barrera, de entrega de desechos a servicios generales, de entrega a la ruta sanitaria, de aplicación de planes de contingencia, de capacitación y de cumplimiento de requisitos legales (9).

El objetivo del programa ambiental es el reducir gradual y constantemente el impacto generado por la disposición inadecuada de desechos sobre el ecosistema, para establecer promedios para el sector industrial y fijar metas cuantificables de reducción en períodos predeterminados de tiempo. La creación y uso de programas de educación ambiental es un elemento clave para la implementación efectiva de un sistema de Gestión ambiental. El programa debe describir como se alcanzarán las metas de la organización, personal responsable, cronograma de actividades, estrategias a seguir, inversiones requeridas y un diseño de sistema de registro (2).
El programa de educación ambiental propuesto, se apoya en la enseñanza problémica que involucra los temas de educación, comunicación, participación e investigación (10). La investigación es un pilar fundamental para el avance y consolidación de la educación ambiental, ya que aporta el conocimiento de las condiciones socio-ambientales del entorno, sus riquezas y potencialidades. La participación ciudadana permite orientar la formación de individuos y colectivos que se involucran en procesos de gestión.

Estas actividades al ser analizadas en cada una de las empresas en la etapa de aplicación y validación del modelo sobre manejo de desechos aportan la claridad que se necesita para dilucidar los puntos críticos de control en el manejo de desechos que van a repercutir en una gestión adecuada, como lo establece la legislación en la política de gestión de residuos promulgada por el Ministerio del Medio Ambiente, Vivienda y Desarrollo (1).

Destacamos como uno de unos puntos críticos de control la capacitación, siendo la educación ambiental a todo nivel pilar para una gestión adecuada de desechos, donde la Universidad debe apoyar y retroalimentar la investigación en aspectos relacionados con la problemática ambiental, estableciendo vínculos entre la industria y la academia.

Con la realización del presente estudio se confirma que no sólo la elaboración de un protocolo o modelo sobre manejo de desechos es fundamental para lograr un 100\% de eficiencia en el manejo y disposición final de desechos, sino que debe complementarse con una capacitación continua y un programa de seguimiento permanente, validación y verificación, que involucre una planeación participativa a largo plazo.

Para finalizar, es importante destacar la importancia de la interrelación de la universidad con instituciones externas, ya que éste no debe ser un ente aislado, sino que debe relacionarse con los demás para mejoramiento de los procesos, de la calidad de vida, contribuyendo a ser más competitivas las instituciones.

Al colaborar en la normalización y elaboración de modelos o protocolos o planes de manejo de desechos internos para cada institución y fortalecer la educación ambiental a través de la generación de procesos participativos con una planeación a largo plazo (11), con seguimiento permanente, en especial de los cambios de actitud, es posible solucionar las diversas problemáticas 
ambientales que produce la contaminación industrial en Colombia, ya que no podemos cambiar el medio ambiente sino cambiamos la cultura.

La divulgación externa del trabajo de investigación nos ha permitido ver que este tema es muy importante en el sector industrial, y que la capacitación dada a partir de este proyecto es necesaria y bien recibida, dejando así una Propuesta de Educación Ambiental obtenida de la experiencia adquirida en la realización del Proyecto sobre Seguimiento, validación y verificación del Modelo sobre manejo de desechos.

El desarrollo de este tipo de proyectos debe seguir en aumento, ya que la universidad debe ser un apoyo para instituciones externas, destacándose en especial el vínculo universidad-empresa, para brindar apoyo, asesoría, investigación, desarrollo e innovación en tecnologías en especial de producción más limpia (PML) (12).

En algunas empresas se observó la cultura del reciclaje como una relación costo/beneficio, o sea una ganancia económica para la empresa y sus trabajadores, lo cual refleja la aplicación de un sistema de gestión ambiental, donde se busca hacer compatible la inversión en la protección del ambiente con la competitividad. El diseño y uso de programas de educación ambiental es un elemento clave para la implementación efectiva de un sistema de gestión ambiental.

\section{Agradecimientos}

Las autoras agradecen el apoyo dado por la Universidad Colegio Mayor de Cundinamarca, por las entidades externas y por los estudiantes de VI semestre del programa de Bacteriología. NOV

\section{Referencias}

1. Ministerio del Medio Ambiente. Políticas para la gestión integral de residuos. Santafé de Bogotá. 1997.

2. Hunt D, Johnson C. Sistemas de Gestión Ambiental. Ed. Mc Graw Hill. Madrid. 1996. 318 p.

3. Sánchez E, Uribe E. Contaminación Industrial en Colombia. DNP-PNUD. Tercer Mundo Editores. Bogotá. 1994. p. 294.

4. Camacho J, Lancheros G. Diagnóstico del manejo actual de los desechos generados en el laboratorio central, área de laboratorios y la IPS de la universidad Colegio Mayor de Cundinamarca con la aplicación a otros laboratorios de las mismas características en Santafé de Bogotá. 2000.

5. Sans R, Ribas J. Ingeniería Ambiental. Contaminación y tratamientos. MARCOMBO. Barcelona, España; BOREAUX. 2002. p147.

6. Delgado F. La relación dialéctica entre la Enseńanza Problémica y un modelo sistémico de la educación. Colección cartillas académicas UNINCCA. Santafé de Bogotá, Colombia. 1997. p. 91.

7. ANTAD. 1993. Guía para el adiestramiento en el manejo y reciclaje de residuos sólidos. Manual Ecológico 3. México. p.42.

8. Ministerio del Medio Ambiente. Política para la Educación Ambiental. Santafé de Bogotá. 1997.

9. Corporación Autónoma Regional de Cundinamarca. Educación para la gestión ambiental. CAR. Bogotá. 1994. p. 80.

10. Medina C. La Enseñanza Problémica - Entre el constructivismo y la educación activa. Ed. Rodríguez Quito Editores. 2da. edición. Santafé de Bogotá, Colombia. 1997. p. 232.

11. Tochobanoglous, G. Gestión Ambiental de residuos sólidos. Mc Graw Hill. España. 2000.

12. Departamento Administrativo del Medio Ambiente. Minimización de la Contaminación Industrial por la promoción de Tecnologías de Producción más limpias en Bogotá. DAMA, JICA. Bogotá. 2000. 